\title{
BMJ Open Association between the recurrence period of acute kidney injury and mortality: a single-centre retrospective observational study in Japan
}

\author{
Keisuke Sako, ${ }^{1}$ Kengo Furuichi, ${ }^{1,2}$ Yuta Yamamura, ${ }^{1}$ Megumi Oshima, ${ }^{1}$ \\ Tadashi Toyama, ${ }^{1}$ Shuichi Kaneko, ${ }^{3}$ Takashi Wada ${ }^{1,4}$
}

To cite: Sako K, Furuichi K, Yamamura Y, et al. Association between the recurrence period of acute kidney injury and mortality: a single-centre retrospective observational study in Japan. BMJ Open 2019;9:e023259. doi:10.1136/ bmjopen-2018-023259

- Additional material is published online only. To view please visit the journal online (http://dx.doi.org/10.1136/ bmjopen-2018-023259)

Received 1 April 2018 Revised 13 March 2019 Accepted 27 March 2019

Check for updates

(c) Author(s) (or their employer(s)) 2019. Re-use permitted under CC BY-NC. No commercial re-use. See rights and permissions. Published by BMJ.

${ }^{1}$ Division of Nephrology, Kanazawa University Hospital, Kanazawa, Ishikawa, Japan ${ }^{2}$ Department of Nephrology, Kanazawa Medical University School of Medicine, Uchinada, Kahoku, Ishikawa, Japan

${ }^{3}$ Department of System Biology, Kanazawa University, Kanazawa, Ishikawa, Japan

${ }^{4}$ Department of Nephrology and Laboratory Medicine, Kanazawa University, Kanazawa, Ishikawa, Japan

Correspondence to

Dr. Kengo Furuichi;

furuichi@kanazawa-med.ac.jp

\section{ABSTRACT}

Objectives Recurrent acute kidney injury (AKI) is a recognised risk factor for mortality. However, it is unclear whether the period until AKI recurrence may have a major factor on patient outcome or not. To explore this issue, we (1) framed the hypothesis that early recurrence increases the risk of mortality and (2) evaluated the prognosis of recurrent AKI cases by setting 21 days as the cut-off period.

Methods All studied cases were admitted and followed up at the Kanazawa University Hospital (Kanazawa, Japan) between 1 November 2006 and 31 October 2007. In total, 21939 patients were retrospectively evaluated in their recurrences of AKI for 2 years and followed up until 31 October 2016. Risks for death were evaluated by the recurrences of AKI (Analysis 1). Patients who developed AKI recurrence before 21 days were defined as the earlyrecurrence group and the remaining cases as the laterecurrence group. Risks for death were evaluated by the two groups (Analysis 2).

Results 510 patients (2.3\%) developed the first AKI. Of these, 151 developed recurrent AKI within 2 years. The number of early-recurrence cases was 44 and that of non-recurrence or late-recurrence was 357. A total of 196 cases $(38.4 \%)$ died, and higher risk for death was observed in the recurrent AKI group (Analysis $1 ; p=0.015$, log-rank test). We found that the rate of all-cause mortality was higher in the early-recurrence group involving 33.8 deaths per 100 person-years, whereas the non-recurrence or late-recurrence group included only 6.2 deaths per 100 person-years (Analysis 2; $p<0.001$, log-rank test).

Conclusions Patients experiencing recurrent AKI before 21 days from the first AKI clearly showed a relatively poor prognosis. Evidently, careful follow-up for at least 21 days after AKI would be highly useful to detect a recurrence event, possibly leading to a better prognosis after AKI.

\section{INTRODUCTION}

Acute kidney injury (AKI) reportedly is a risk factor for end-stage kidney disease (ESKD) and all-cause mortality. ${ }^{1-4}$ Although shortterm mortality and the recovery of kidney function after AKI are serious and critical issues for each patient, recent studies have
Strengths and limitations of this study

- Few studies have examined the risks related to short-term recurrent acute kidney injury (AKI) and prognosis.

- The mean follow-up period was 2.9 years, up to 10 years.

- For detailed analysis of the risks related to the AKI recurrence, recurrent AKI was classified as early-recurrent and late-recurrent AKI.

- It was unknown whether the observed AKI was a cause of death or a complication not directly related to the death.

- A single-centre study might result in low power in the analysis.

revealed that AKI also affects long-term mortality for more than several months or even several years. ${ }^{5-7}$ Therefore, an understanding of the risks of long-term mortality can potentially prevent an inaccurate prognosis of kidney function and mortality after AKI.

A key prognostic factor for mortality after AKI is the severity of the event itself. ${ }^{8-11}$ In addition, recurrent $\mathrm{AKI}$ is reported to be a risk factor for (1) chronic kidney disease (CKD) in individuals with transplanted kidneys ${ }^{12}$ and (2) ESKD progression and mortality. Moreover, a recent study revealed that the risk of a second AKI is diverse among AKI survivors and that the recurrence of AKI is common. ${ }^{13}$ Prevention of recurrent AKI would help reduce long-term kidney dysfunction and mortality, suggesting the need for sustained and careful clinical observation after AKI.

The optimal method of care for patients after AKI has been under investigation for some time; at present, a completely defined optimal protocol does not exist. Certainly, close observation of patients after AKI may provide an opportunity to prevent long-term 


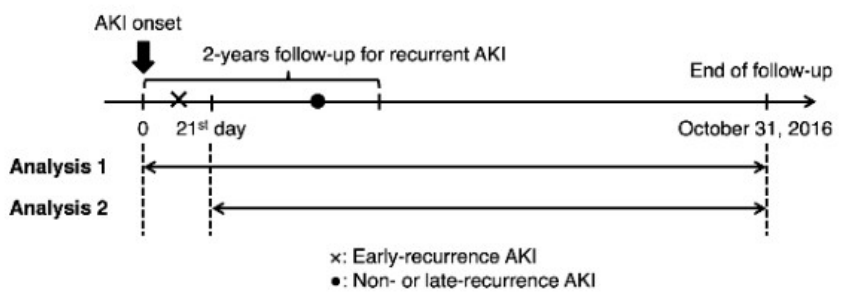

Figure 1 Study design. Patients were included and followed up from acute kidney injury (AKI) onset to 31 October 2016. In Analysis 2, patients were included and followed from the 21st day of first AKI onset to 31 October 2016.

kidney dysfunction and its sequelae. ${ }^{14}$ In particular, a referral to a nephrologist may benefit dialysis-requiring survivors of an AKI event. ${ }^{15}$ However, the optimal monitoring period for patients with AKI after their kidney injury remains unclear.

In this study, we investigated the clinical features of patients with recurrent AKI and evaluated the risk factors for mortality and their relationship with the recurrence period of AKI.

\section{METHODS}

\section{Study population and setting}

For this study, an inclusion criterion was that all of the cases were admitted and followed up at the Kanazawa University Hospital (Kanazawa, Japan) between 1 November 2006 and 31 October 2007. In total, 123493 blood samples of 21939 patients were collected and retrospectively evaluated (figure 1). After excluding maintenance dialysis

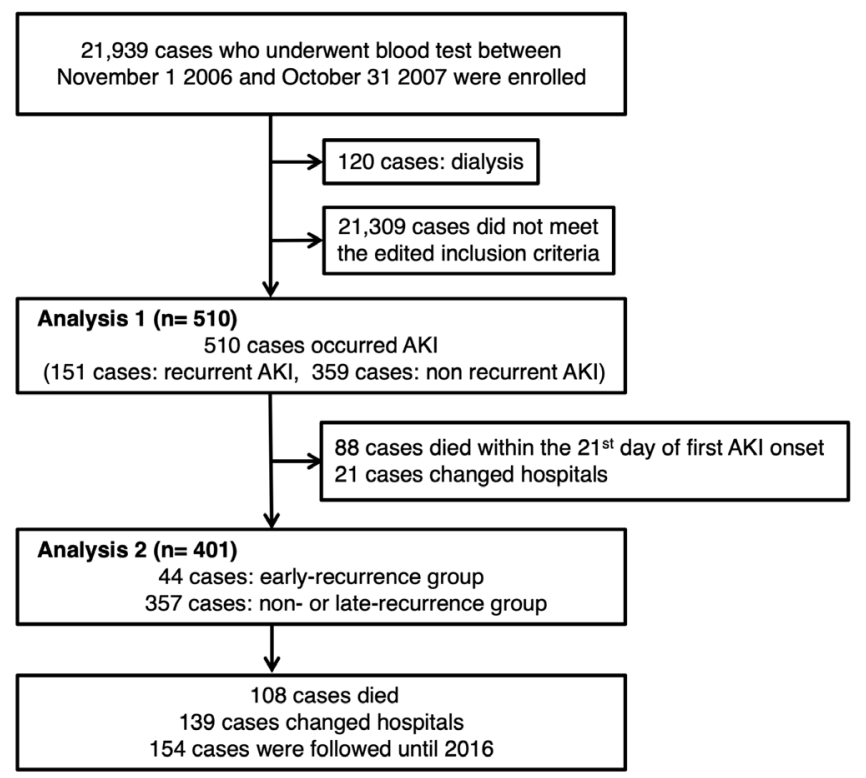

Figure 2 Flow chart of the study. In total, 21309 cases were excluded because they did not meet the AKI (acute kidney injury) criteria defined by KDIGO (Kidney Disease Improving Global Outcomes). Cases were included in this study at the occurrence of the first AKI. A total of 160 patients changed hospitals within 10 years; thus, their outcome was unknown. case, 510 patients $(2.3 \%)$ with AKI events were finally detected. Their medical records were collected from the electronic medical records, and these data included the following: age, sex, serum creatinine (SCr) level, admission to the intensive care unit (ICU), cause of first AKI, comorbidity, medication and mortality. While follow-up data were collected at 10 years (until 31 October 2016), recurrent AKI events were detected within 2 years from the first onset of AKI (figure 1). The primary endpoint in this study was death.

Information about this study was announced to potential candidates on the Kanazawa University Hospital website instead of obtaining informed consent from each patient. All analyses were performed on the condition of anonymity, and the patients could withdraw from the study at any point.

\section{Patient and public involvement}

The patients were not involved in the design of, recruitment for, or conduct of this study. Development of the outcome measures was not informed by the priorities, experiences or preferences of the patients. Contributions to this study made by the many participants are deeply appreciated, and the results of this joint effort will be publicly available to the patients via this publication.

\section{Definition of AKI and recurrent AKI}

In accordance with the KDIGO criteria, ${ }^{16}$ AKI was defined as an increase in SCr level by $\geq 0.3 \mathrm{mg} / \mathrm{dL}$ within 48 hours from a baseline SCr measurement or an increase in $\mathrm{SCr}$ level by $\geq 50 \%$ within the prior 7 days. Consistent with a previous investigation, ${ }^{17}$ AKI was defined only as an increase in SCr level in our study. Baseline SCr level was defined by the most recently available value before AKI. ${ }^{18}$ Further, recurrent AKI was defined as the re-increase of SCr levels from the lowest SCr level after the previous AKI event, following partial or complete recovery. To avoid misclassification, a decrease in SCr level from their maximal value at least twice before a recurrent AKI was considered to have occurred. In particular, increases in SCr level after renal replacement therapy withdrawal were not defined as AKI recurrences. Cases that developed recurrent AKI before 21 days were defined as the early-recurrence group, and all others that developed recurrent AKI after 21 days within the 2-year observation period were placed in the late-recurrence group. In this study, 21 days was used as an assessment period for recurrent AKI based on the ROC curve (AUC=0.581). We conducted two analyses. Recurrent AKI was compared with non-recurrent AKI (Analysis 1); early-recurrence AKI was compared with non-recurrence or late-recurrence AKI (Analysis 2).

\section{Statistical analysis}

The data were prepared as percentages, mean values $\pm \mathrm{SD}$, medians (range) or interquartile rankings (IQR). Categorical variables were analysed using $\chi^{2}$ or Fisher's exact test, whereas continuous variables were compared using the independent t-test. Cumulative survival was estimated 
Table 1 Baseline characteristics of study population

\begin{tabular}{|c|c|c|c|}
\hline & Non-recurrent AKI ( $n=359)$ & $\begin{array}{l}\text { Recurrent AKI } \\
(n=151)\end{array}$ & $P$ value \\
\hline Age (years), median (IQR) & $64(53-73)$ & $60(44-73)$ & 0.135 \\
\hline Sex (male) & 220 (61\%) & $100(66 \%)$ & 0.292 \\
\hline $\begin{array}{l}\text { Baseline SCr (mg/dL), median } \\
\text { (IQR) }\end{array}$ & $0.75(0.56-1.10)$ & $0.88(0.60-1.16)$ & 0.377 \\
\hline \multicolumn{4}{|l|}{ AKI severity (KDIGO) } \\
\hline Stage I & $222(62 \%)$ & $99(66 \%)$ & 0.010 \\
\hline Stage II & $64(18 \%)$ & $37(25 \%)$ & \\
\hline Stage III & $73(20 \%)$ & $15(10 \%)$ & \\
\hline Admission to ICU at first AKI & $74(21 \%)$ & $23(15 \%)$ & 0.157 \\
\hline \multicolumn{4}{|l|}{ Comorbidities } \\
\hline Hypertension & $135(38 \%)$ & $62(41 \%)$ & 0.464 \\
\hline Diabetes mellitus & $80(22 \%)$ & 45 (30\%) & 0.072 \\
\hline Coronary artery disease & $41(11 \%)$ & $25(17 \%)$ & 0.115 \\
\hline $\mathrm{CHF}$ & $72(20 \%)$ & $40(27 \%)$ & 0.109 \\
\hline Advanced LD & $31(9 \%)$ & $26(17 \%)$ & 0.005 \\
\hline Malignancy & $158(44 \%)$ & $94(62 \%)$ & $<0.001$ \\
\hline \multicolumn{4}{|l|}{ Drug } \\
\hline Diuretic & $143(40 \%)$ & 79 (52\%) & 0.009 \\
\hline ACEi/ARB & $79(22 \%)$ & $37(24 \%)$ & 0.539 \\
\hline Deaths & $116(32 \%)$ & $80(53 \%)$ & $<0.001$ \\
\hline
\end{tabular}

II receptor blocker.

Values for continuous variables, as mean \pm SD deviation.

Conversion factors for serum creatinine in $\mathrm{mg} / \mathrm{dL}$ to $\mu \mathrm{mol} / \mathrm{L}, \times 88.4$.

ACEi, ACE inhibitor; AKI, acute kidney injury; ARB, angiotensin; CHF, chronic heart failure; ICU, intensive care unit; KDIGO, Kidney Disease Improving Global Outcomes; LD, liver disease; SCr, serum creatinine.

by drawing Kaplan-Meier survival curves, and survival rates were compared using the log-rank test. In Analysis 2, patients were followed from the 21st day of the onset of first AKI (figure 1). To be classified as early-recurrence or late-recurrence AKI, the patients had to survive at least for 21 days from the onset of first AKI; hence, in Analysis 2 , considering time bias, we excluded patients who died before the 21st day. A p value of $<0.05$ was considered to indicate a statistically significant difference. SPSS Statistics V.25.0 (Armonk, New York, USA: IBM Corp) was used for the statistical analysis.

\section{RESULTS}

Selection of all 510 patients included in the study was shown in (figure 2). Baseline characteristics of the study population are presented in table 1 . While 359 cases $(70.4 \%)$ involved one AKI (non-recurrent AKI), $108(21.2 \%)$ involved two such events. Further, 43 cases (8.4\%) showed recurrent AKI >3 times within our 2-year observation period. The median period of AKI recurrence was 36 days; remarkably, there were no significant differences in terms of age, sex and baseline SCr levels between the groups. The proportion of the patients with
AKI stage III in the non-recurrent AKI group (20\%) was significantly higher than that in the recurrent AKI group $(10 \%)(\mathrm{p}=0.010)$, whereas advanced liver disease, malignancy and use of diuretics were more frequent in the recurrent AKI group.

The various causes of AKI for our particular patient group are summarised in table 2. Notably, the proportion of volume depletion $(31.5 \%$ vs $21.2 \%, \mathrm{p}=0.019)$ and cardiac surgery $(7.5 \%$ vs $2.6 \%, \mathrm{p}=0.036)$ cases in the non-recurrent AKI group was significantly higher than that in the recurrent AKI group. In contrast, the proportion of chronic heart failure (4.7 vs $10.6 \%, \mathrm{p}=0.014)$ and chemotherapy $(8.9 \%$ vs $24.5 \%, \mathrm{p}<0.001)$ causes was significantly higher in the recurrent AKI group.

\section{Analysis 1}

In total, 196 cases (38.4\%) died during our 10-year observation period. The death group was clearly older than its surviving counterpart (median: 62 (49-73) vs 64 (53-74) years, $\mathrm{p}=0.041$ ), and the stage of AKI was significantly higher in the death group ( $\mathrm{p}<0.001$, data not shown). The proportion of the patients with AKI stage III was significantly higher in the non-recurrent AKI group $(p=0.010)$, but the mortality rate was significantly higher 
Table 2 Causes of first AKI

\begin{tabular}{lllll}
\hline & Total $(\mathbf{n = 5 1 0 )}$ & $\begin{array}{l}\text { Non-recurrent AKI } \\
(\mathbf{n = 3 5 9 )}\end{array}$ & $\begin{array}{l}\text { Recurrent AKI } \\
(\mathbf{n = 1 5 1 )}\end{array}$ & P value \\
\hline Volume depletion & $145(28.4 \%)$ & $113(31.5 \%)$ & $32(21.2 \%)$ & 0.019 \\
\hline Sepsis & $71(13.9 \%)$ & $46(12.8 \%)$ & $25(16.6 \%)$ & 0.265 \\
\hline Chemotherapy & $69(13.5 \%)$ & $32(8.9 \%)$ & $37(24.5 \%)$ & $<0.001$ \\
\hline Abdominal surgery & $47(9.2 \%)$ & $34(9.5 \%)$ & $13(8.6 \%)$ & 0.759 \\
\hline Malignancy & $45(8.8 \%)$ & $35(9.7 \%)$ & $10(6.6 \%)$ & 0.256 \\
\hline CHF & $33(6.5 \%)$ & $17(4.7 \%)$ & $16(10.6 \%)$ & 0.014 \\
\hline Cardiac surgery & $31(6.1 \%)$ & $27(7.5 \%)$ & $4(2.6 \%)$ & 0.036 \\
\hline Advanced LD & $16(3.1 \%)$ & $10(2.8 \%)$ & $6(4.0 \%)$ & 0.482 \\
\hline Vascular surgery & $11(2.2 \%)$ & $10(2.8 \%)$ & $1(0.7 \%)$ & 0.132 \\
\hline $\begin{array}{l}\text { Acute coronary } \\
\text { syndrome }\end{array}$ & $3(0.6 \%)$ & $3(0.8 \%)$ & $0(0 \%)$ & 0.260 \\
Others & $39(7.6 \%)$ & $32(8.9 \%)$ & $7(4.6 \%)$ & 0.097 \\
\hline
\end{tabular}

AKI, acute kidney injury; CHF, chronic heart failure; LD, liver disease.

in the recurrent AKI $(53.0 \%)$ compared with the non-recurrent AKI group (32.3\%) $(\mathrm{p}<0.001)$. The recurrent AKI patients also showed different survival curves from the non-recurrent AKI group (figure 3; $\mathrm{p}=0.015$, log-rank test) because, somewhat surprisingly, within 5 months, the non-recurrent AKI group showed a poorer prognosis than their recurrent AKI counterparts. However, in contrast to the non-recurrent AKI group, the actual survival rate of the recurrent AKI group gradually decreased.

\section{Analysis 2}

We focused on the observation of patients with recurrent AKI based on the hypothesis that patients with early recurrent AKI do worse. Clinical backgrounds of the early-recurrence group and non-recurrence or late- recurrence

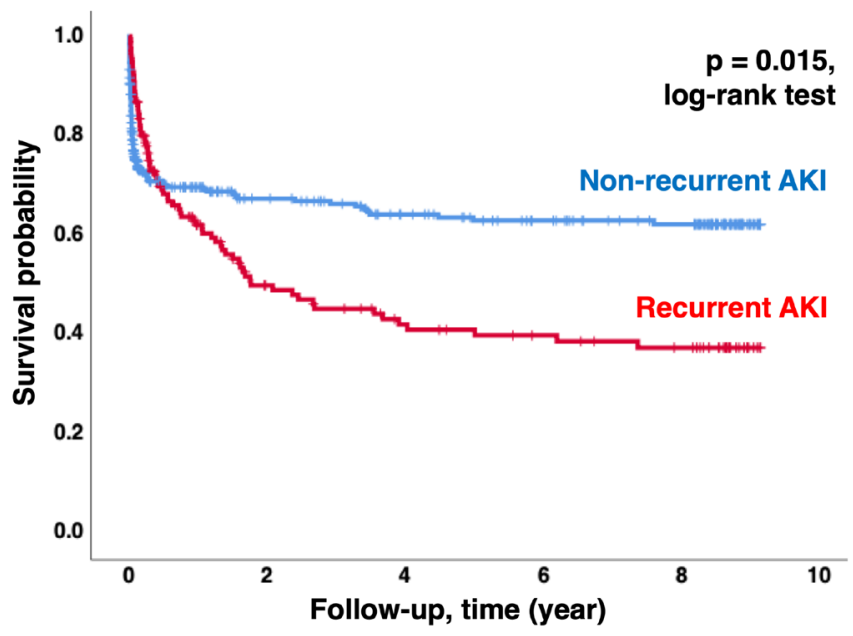

Figure 3 Survival curve over 10 years (Analysis 1). Survival curve of the non-recurrent acute kidney injury (AKI) and recurrent AKI groups over 10 years according to the KaplanMeier method. Differences between the groups were compared by the log-rank test. Blue line, non-recurrent AKI group $(n=359)$; red line, recurrent $A K I$ group $(n=151)$. groups are presented in table 3. Of all 401 patients included in Analysis 2 (figure 2), 44 cases were early-recurrence group, and 357 cases were non-recurrence or late-recurrence group. In the early-recurrence group, 41 cases (93.2\%) had next AKI before discharge form the hospital. In the late-recurrence group, 43 cases $(44.8 \%)$ had next AKI before discharge from the hospital. The late-recurence were lower frequency than early-recurrence $(p<0.001)$. The survival curve analysis of cases with recurrent AKI is shown in figure 4. The mean survival time was $76.3 \pm 2.6$ months (95\% CI 71.1 to 81.4 ). As expected, the mortality rate was significantly higher in the early-recurrence group compared with non-recurrence or late-recurrence AKI ( $<<0.001, \log$-rank test); in particular, the early-recurrence group had 33.8 deaths per 100 person-years, whereas the non-recurrence or late- recurrence group had only 6.2 deaths per 100 person-years. These results suggested that the early-recurrent AKI was leading to poor prognosis. The survival curve analysis of each group is shown in online supplementary figure 1 .

The proportion of cases with using diuretics ( $71 \%$ vs $40 \%$, $\mathrm{p}<0.001)$ in the early group was significantly higher than that in non or late group (table 3). Howeve, there was no significant difference in cause of the first AKI between the two groups (table 4 ).

\section{DISCUSSION}

This study indicated the importance of observing patients with AKI for at least 21 days to best detect recurrent AKI with poor prognosis. Moreover, as expected, the mortality rate in the recurrent AKI group was higher than that for their AKI counterparts. In particular, an early recurrence of AKI indicated a particularly poor prognosis. The findings of this retrospective study indicate that AKI cases should be monitored for several weeks to prevent AKI recurrence and improve the mortality rate after AKI. 
Table 3 Characteristics of recurrent AKI patients

\begin{tabular}{|c|c|c|c|}
\hline & $\begin{array}{l}\text { Early recurrence } \\
(n=44)\end{array}$ & $\begin{array}{l}\text { Non-recurrence or } \\
\text { late recurrence } \\
(n=357)\end{array}$ & $P$ value \\
\hline Age (years), median (IQR) & $54(43-74)$ & $58(51-72)$ & 0.316 \\
\hline Sex (male) & $22(50 \%)$ & 227 (64\%) & 0.080 \\
\hline $\begin{array}{l}\text { Baseline SCr (mg/dL), median } \\
\text { (IQR) }\end{array}$ & $0.95(0.52-1.14)$ & $1.01(0.79-1.15)$ & 0.681 \\
\hline stage II & $9(20 \%)$ & $60(17 \%)$ & \\
\hline stage III & $3(7 \%)$ & $54(15 \%)$ & \\
\hline Admission to ICU at first AKI & $10(23 \%)$ & $46(13 \%)$ & 0.076 \\
\hline $\begin{array}{l}\text { Comorbidities } \\
\text { Hypertension }\end{array}$ & $19(43 \%)$ & $140(39 \%)$ & 0.612 \\
\hline Advanced LD & $6(14 \%)$ & $36(10 \%)$ & 0.468 \\
\hline Malignancy & $22(50 \%)$ & $174(49 \%)$ & 0.875 \\
\hline $\begin{array}{l}\text { Drug } \\
\text { Diuretic }\end{array}$ & $31(71 \%)$ & 143 (40\%) & $<0.001$ \\
\hline ACEi/ARB & $11(25 \%)$ & $95(27 \%)$ & 0.819 \\
\hline $\begin{array}{l}\text { Deaths after } 21^{\text {st }} \text { days from the } \\
\text { first AKI }\end{array}$ & $26(59 \%)$ & $82(23 \%)$ & $<0.001$ \\
\hline
\end{tabular}

II receptor blocker.

Values for continuous variables, as mean \pm SD deviation.

Conversion factors for serum creatinine in $\mathrm{mg} / \mathrm{dL}$ to $\mu \mathrm{mol} / \mathrm{L}, \times 88.4$.

ACEi, ACE inhibitor; AKI, acute kidney injury; ARB, angiotensin; CHF, chronic heart failure; ICU, intensive care unit; KDIGO, Kidney Disease Improving Global Outcomes; LD, liver disease; SCr, serum creatinine.

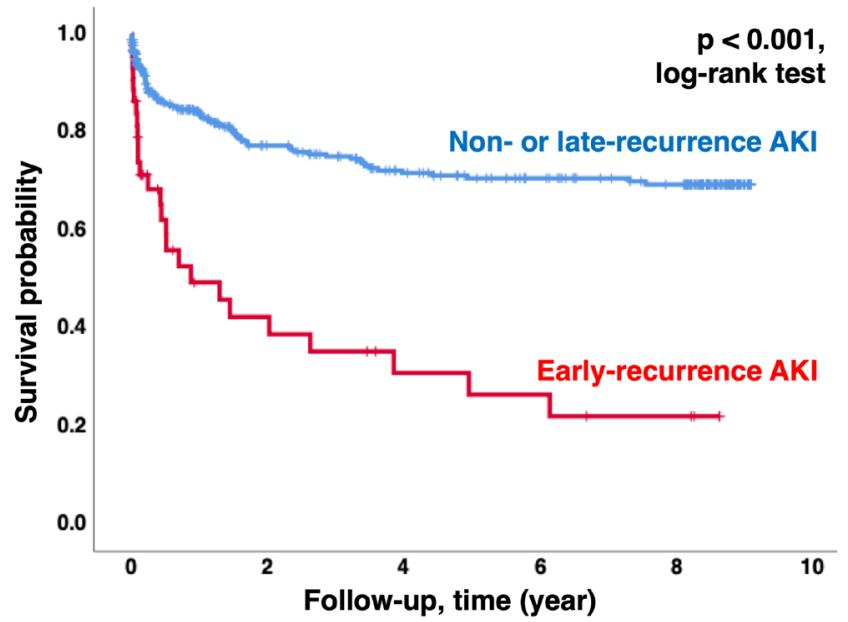

Figure 4 Survival curve over 10 years (Analysis 2). Survival curve of the early-recurrence group and non-recurrence or late-recurrence groups over 10 years according to the Kaplan-Meier method. Follow-up was started from the 21st day of the onset of first acute kidney injury (AKI). Differences between the groups were compared by the log-rank test. Blue line, non-recurrence or late-recurrence group $(n=357)$; red line, early-recurrence group $(n=44)$.
Somewhat paradoxically, within 5 months, the non-recurrent AKI group (no recurrence within 2years) showed a poorer prognosis than the recurrent AKI group. In remarkable contrast, the mortality rate of the recurrent AKI group actually decreased over 10 years. In general, the mortality rate after AKI was reported to be poor during the initial several weeks, but the rate subsequently showed almost no change after the initial period. ${ }^{19-21}$ Similar findings have been reported that the poor mortality in the early phase after AKI lasted approximately 42 days, ${ }^{22}$ and our data show similar results. However, the recurrent AKI group showed different survival curves from the non-recurrent AKI group. Sawhney et al reported that patients with prior AKI episodes had similar early mortality, but greater mortality from 6 months onward than patients without prior episodes. ${ }^{21}$ It should be noted that the recurrent AKI episodes gradually worsened the mortality as compared with the non-recurrent AKI episodes. These findings would again indicate that recurrent AKI is not a simple repetition of AKI and that careful observation for several weeks after AKI is beneficial to improve the accuracy of a prognosis after an AKI event. However, there have been no reports on period for recurrent AKI and mortality. 
Table 4 There was no difference in cause of first AKI between two groups

\begin{tabular}{llll}
\hline & $\begin{array}{l}\text { Early recurrence } \\
(\mathbf{n}=\mathbf{4 4 )}\end{array}$ & $\begin{array}{l}\text { Non-recurrence or } \\
\text { late recurrence } \mathbf{( n = 3 5 7 )}\end{array}$ & P value \\
\hline Volume depletion & $12(27.3 \%)$ & $94(26.3 \%)$ & 0.894 \\
Sepsis & $7(15.9 \%)$ & $52(14.6 \%)$ & 0.812 \\
Chemotherapy & $11(25.0 \%)$ & $53(14.8 \%)$ & 0.083 \\
\hline Abdominal surgery & $4(9.1 \%)$ & $42(11.8 \%)$ & 0.599 \\
\hline Malignancy & $1(2.3 \%)$ & $20(5.6 \%)$ & 0.350 \\
\hline CHF & $5(11.4 \%)$ & $19(5.3 \%)$ & 0.111 \\
Cardiac surgery & $1(2.3 \%)$ & $26(7.3 \%)$ & 0.211 \\
Advanced LD & $2(4.5 \%)$ & $9(2.5 \%)$ & 0.438 \\
Vascular surgery & $1(2.3 \%)$ & $7(1.7 \%)$ & 0.889 \\
\hline Acute coronary syndrome & $0(0 \%)$ & $3(0.8 \%)$ & 0.542 \\
\hline Others & $0(0 \%)$ & $32(9.0 \%)$ & 0.038 \\
\hline
\end{tabular}

$\mathrm{AKI}$, acute kidney injury; CHF, chronic heart failure; LD, liver disease.

The early-recurrence group showed a significantly poorer prognosis than their non-recurrence or late-recurrence counterparts. There was a slight difference (regarding use of a diuretic) in terms of the clinical baseline characteristics and causes of the first AKI between the early-recurrence and late-recurrence groups, indicating that it is difficult to make an accurate prognosis during the first AKI. As further examples of prediction difficulty, advanced age and low estimated glomerular filtration rate were reported to be risk factors for AKI, ${ }^{13} 23$ and the intensity of AKI was reported to be a risk factor for mortality and kidney function after AKI. ${ }^{8-11}$ However, these elements were not reliable predictors of recurrent AKI in our study. Such findings indicate that recurrent AKI is not a simple repetition of AKI. In some contrast, a recent study straightforwardly indicated that patients with a longer AKI duration, congestive heart failure, decompensated advanced liver disease, cancer with or without chemotherapy, acute coronary syndrome or volume depletion were at the highest risk for being hospitalised with recurrent AKI. ${ }^{13}$ Similar findings were also previously reported. ${ }^{24-26}$ Recently, Rodriguez et al reported a singlecentre retrospective observational study in Spain about recurrent AKI. As in previous reports and our results, they showed poor prognosis in the recurrent AKI group (HR: 4.5 (95\% CI 2.7 to 7.5$) \mathrm{p}<0.001)$. However, in this report, the relationship between time to recurrence and prognosis was not shown. Our aim is to show the relationship between time to recurrence and prognosis. We believe that the paper does not compete particularly because the paper did not indicate it. Our report has originality in this point. ${ }^{27}$ In our study, we did have the clear result that approximately $50 \%$ of the patients died of a malignant tumour and 20\% developed cardiovascular disease after AKI. Therefore, the kidney function of cases with these conditions should be monitored after AKI. However, it remains unclear how often recurrent AKI occurred in these cases and how a poor prognosis of early recurrent
AKI could have been predicted in the high-risk group. In the future, a prospective study with a large number of cases and use of sensitive biomarkers would be necessary to detect recurrent AKI.

\section{Limitations}

This study has certain limitations. First, the data originated from a single university hospital in Kanazawa. The power to lead these results might be small. Second, this was a retrospective observational study. Because blood sampling is dependent on clinical requirements, the interval of blood sampling varied according to each case. Therefore, we may have missed actual AKI and recurrent AKI cases. Third, it was difficult to judge whether AKI was a direct cause of death or an incidental factor because we could use only the medical records in our hospital. We could not use the competing risk model to calculate the HR. Lastly, there remains incomplete evidence that precisely 21 days is the optimal period to monitor for the early recurrence in this study. Additional multicentre and prospective research is required to overcome these limitations.

\section{CONCLUSIONS}

The mortality rate in the recurrent AKI group was found to be higher than in the non-recurrent AKI group; in particular, the short-term recurrence of AKI indicated an especially poor prognosis.

To prevent AKI recurrence and decrease mortality rate after AKI, AKI cases should be carefully monitored for several weeks after the injury.

Acknowledgements The authors thank all the individuals concerned in this study. Contributors Research idea and study design: KF and TW; data acquisition: YY, $\mathrm{MO}$ and KS; data analysis/interpretation: YY, MO and KS; and statistical analysis: TT and KS; supervision or mentorship: KF, SK and TW. Each author contributed important intellectual content during the manuscript drafting or revision and accepts accountability for the overall work by ensuring that questions pertaining to 
the accuracy or integrity of any portion of the work are appropriately investigated and resolved. KF assures that this study has been reported with reliability, accuracy and transparency.

Funding The authors have not declared a specific grant for this research from any funding agency in the public, commercial or not-for-profit sectors.

\section{Competing interests None declare.}

Patient consent for publication Not Obtained.

Ethics approval The Ethics Committee of the Kanazawa University Hospital (Kanazawa, Japan) approved this study (Approval No. 907-4), and it was conducted in accordance with the Declaration of Helsinki.

Provenance and peer review Not commissioned; externally peer reviewed.

Data sharing statement Data will be shared by going through the procedures of the Ethics Committee of Kanazawa University Hospital.

Open access This is an open access article distributed in accordance with the Creative Commons Attribution Non Commercial (CC BY-NC 4.0) license, which permits others to distribute, remix, adapt, build upon this work non-commercially, and license their derivative works on different terms, provided the original work is properly cited, appropriate credit is given, any changes made indicated, and the use is non-commercial. See: http://creativecommons.org/licenses/by-nc/4.0/.

\section{REFERENCES}

1. Chertow GM, Levy EM, Hammermeister KE, et al. Independent association between acute renal failure and mortality following cardiac surgery. Am J Med 1998;104:343-8.

2. Chertow GM, Burdick E, Honour M, et al. Acute kidney injury, mortality, length of stay, and costs in hospitalized patients. J Am Soc Nephrol 2005;16:3365-70.

3. Thakar CV, Christianson A, Freyberg R, et al. Incidence and outcomes of acute kidney injury in intensive care units: a Veterans Administration study. Crit Care Med 2009;37:2552-8.

4. Thakar CV. Perioperative acute kidney injury. Adv Chronic Kidney Dis 2013;20:67-75

5. Lafrance JP, Miller DR. Acute kidney injury associates with increased long-term mortality. J Am Soc Nephrol 2010;21:345-52.

6. Wu VC, Huang TM, Lai CF, et al. Acute-on-chronic kidney injury at hospital discharge is associated with long-term dialysis and mortality. Kidney Int 2011;80:1222-30.

7. Jones J, Holmen J, De Graauw J, et al. Association of complete recovery from acute kidney injury with incident CKD stage 3 and allcause mortality. Am J Kidney Dis 2012;60:402-8.

8. Ricci Z, Cruz D, Ronco C. The RIFLE criteria and mortality in acute kidney injury: A systematic review. Kidney Int 2008;73:538-46.

9. Lopes JA, Fernandes P, Jorge S, et al. Acute kidney injury in intensive care unit patients: a comparison between the RIFLE and the Acute Kidney Injury Network classifications. Crit Care 2008;12:R110.
10. Joannidis M, Metnitz B, Bauer $\mathrm{P}$, et al. Acute kidney injury in critically ill patients classified by AKIN versus RIFLE using the SAPS 3 database. Intensive Care Med 2009;35:1692-702.

11. Srisawat N, Hoste EE, Kellum JA. Modern classification of acute kidney injury. Blood Purif 2010;29:300-7.

12. Bardak S, Turgutalp K, Türkegün $\mathrm{M}$, et al. Recurrent Acute Kidney Injury in Renal Transplant Patients: A Single-Center Study. Transplant Proc 2015;47:1437-41.

13. Siew ED, Parr SK, Abdel-Kader K, et al. Predictors of Recurrent AKI. J Am Soc Nephrol 2016;27:1190-200.

14. Goldstein SL, Jaber BL, Faubel S, et al. AKI transition of care: a potential opportunity to detect and prevent CKD. Clin J Am Soc Nephrol 2013;8:476-83

15. Harel Z, Wald R, Bargman JM, et al. Nephrologist follow-up improves all-cause mortality of severe acute kidney injury survivors. Kidney Int 2013;83:901-8.

16. Section 2: AKI Definition. Kidney Int Suppl (2011). , 2012:2, 19-36.

17. Barrantes F, Tian J, Vazquez R, et al. Acute kidney injury criteria predict outcomes of critically ill patients. Crit Care Med 2008;36:1397-403

18. Rodrigo E, Suberviola B, Santibáñez M, et al. Association between recurrence of acute kidney injury and mortality in intensive care unit patients with severe sepsis. J Intensive Care 2017;5:5-28.

19. Ronco C, Belomo R, Homel P, et al. Effects of different doses in continuous veno-venous haemofiltration on outcomes of acute renal failure: a prospective randomised trial. Edtna Erca J 2002;Suppl 2:7-12.

20. Gastaldello K, Melot C, Kahn RJ, et al. Comparison of cellulose diacetate and polysulfone membranes in the outcome of acute rena failure. A prospective randomized study. Nephrol Dial Transplant 2000;15:224-30.

21. Sawhney S, Marks A, Fluck N, et al. Intermediate and Long-term Outcomes of Survivors of Acute Kidney Injury Episodes: A Large Population-Based Cohort Study. Am J Kidney Dis 2017;69:18-28.

22. Zhang JH, Palevsky PM, Chertow GM, et al. Piecewise analysis of patient survival after onset of AKI. Clin J Am Soc Nephrol 2013;8:1679-84

23. Collins AJ, Foley RN, Chavers B, et al. US Renal Data System 2013 Annual Data Report. Am J Kidney Dis 2014;63(1 Suppl):A7.

24. Campbell GA, Hu D, Okusa MD. Acute kidney injury in the cance patient. Adv Chronic Kidney Dis 2014;21:64-71.

25. Selby NM, Kolhe NV, Mclntyre CW, et al. Defining the cause of death in hospitalised patients with acute kidney injury. PLoS One 2012; 7:e48580.

26. Bagshaw SM, Laupland KB, Doig CJ, et al. Prognosis for long-term survival and renal recovery in critically ill patients with severe acute renal failure: a population-based study. Crit Care 2005:9:R700-9.

27. Rodríguez E, Arias-Cabrales C, Bermejo S, et al. Impact of Recurrent Acute Kidney Injury on Patient Outcomes. Kidney Blood Press Res 2018;43:34-44. 